Article

\title{
Effect of Fabrication Method on Fracture Strength of Provisional Implant-Supported Fixed Dental Prostheses
}

\author{
Kelly M. Suralik ${ }^{1} \mathbb{D}$, Jie Sun ${ }^{1}$, Chia-Yu Chen ${ }^{2}$ and Sang J. Lee ${ }^{1, * \mathbb{C}}$ \\ 1 Department of Restorative Dentistry and Biomaterial Sciences, 188 Longwood Avenue, Harvard School of \\ Dental Medicine, Boston, MA 02120, USA; ksuralik@gmail.com (K.M.S.); jiejsun@gmail.com (J.S.) \\ 2 Department of Oral Medicine, Infection and Immunity, 188 Longwood Avenue, Harvard School of Dental \\ Medicine, Boston, MA 02120, USA; chia-yu_chen@hsdm.harvard.edu \\ * Correspondence: sang_lee@hsdm.harvard.edu; Tel.: +1-617-432-3064
}

Received: 30 September 2020; Accepted: 1 November 2020; Published: 4 November 2020

check for updates

\begin{abstract}
There has been an increase in utilizing 3D printers in dental restorations. The purpose of the study is to compare mechanical properties of 3D-printed prostheses to those of self-cured and/or computer-aided design-computer-aided manufacturing (CAD-CAM) restorations. A metal master typodont was prepared for the mandibular left sextant with implant analogs embedded at the first premolar and first molar positions with a missing second premolar. Three-unit provisional fixed dental prosthesis (FDP) was designed utilizing the 3Shape tooth library and forty-five uniform specimens were fabricated with different materials: self-cured poly (methyl methacrylate) (PMMA) $(\mathrm{N}=15)$, milled PMMA CAD-CAM blocks $(\mathrm{N}=15)$ and 3D-printed resin $(\mathrm{N}=15)$. All specimens were tested using an Instron machine at a crosshead speed of $0.5 \mathrm{~mm} / \mathrm{min}$ by an axial load on the occlusal surface of the second premolar pontic site. Statistical analysis was completed with Shapiro-Wilk, ANOVA and Tukey post-hoc tests. Mean fracture force was $300.61 \mathrm{~N}, 294.64 \mathrm{~N}$ and $408.49 \mathrm{~N}$ for self-cured PMMA, milled PMMA and 3D-printed resin, respectively. Mean force at FDP fracture of 3D-printed resin was significantly greater than the mean fracture force of either self-cured $(p=0.016$, 95\% CI [17.86, 197.91]) or milled ( $p=0.010,95 \%$ CI [23.83, 203.88]) PMMA.
\end{abstract}

Keywords: fracture strength; 3D-printed resin; provisional restorations

\section{Introduction}

Provisional restorations are essential for treatment, providing protection to prepared tooth structure, pulp and surrounding periodontal tissues [1-5]. Provisional prostheses help prevent caries and migration of abutments and allow clinicians to evaluate parallelism, function, esthetics and phonetics [1-3,5-8]. Patient goals of maintaining function and esthetics are also achieved with temporary restorations [1]. Desirable characteristics of provisional restorations include strength, retention, marginal accuracy, dimensional stability, color stability, ease of cleaning and ease of fabrication and repair [2-5,7-10]. While fundamental to prosthodontic care, provisional restorations can also assist in periodontal, orthodontic, occlusion and implant therapies [3].

Polymeric resin is widely-used for provisional restorations. Poly(methyl methacrylate) (PMMA) is a popular material for provisional restorations due to high strength, color stability and ease of repair [11]. Unfortunately, chairside fabrication with self-cured PMMA results in an exothermic reaction which may irritate pulpal tissues and polymerization shrinkage which may cause distortion of the restoration [3]. Digital technology and computer-aided design-computer-aided manufacturing (CAD-CAM) have expedited delivery of provisional restorations by reducing chairside and laboratory 
time [4]. CAD-CAM of PMMA provisional prostheses avoids heat of polymerization and shrinkage associated with self-cured PMMA. The advantages of provisional restorations via CAD-CAM process over conventional curing include increased durability [1,3,4,12,13], improved fit $[3,4]$ and color stability [4]. However, fabrication with milling also has disadvantages: material waste, introduction of microcracks and limited reproduction of surface details depending on the size of the milling instrumentation [14].

With the increasing availability of 3D printers, digitally-designed provisional restorations can be printed in addition to being milled. Additive manufacturing creates less raw material waste, reduces manufacturing time and allows for mass production as compared to milling $[6,14,15]$ but 3D-printed structures have limited shade selection, display a less finished surface due to anisotropy and the staircase effect and require expensive post-processing for ceramic [6,14]. Recently, investigators found that the accuracy of dental restorations fabricated by additive manufacturing methods is higher than that of subtractive methods [16]. The internal and marginal fits of 3D-printed provisional single-unit restorations were found to be comparable to those of crowns fabricated using a milling technique, thus making the 3D printing of provisional restorations a viable alternative [17]. Tahayeri et al. concluded that 3D-printed samples withstand peak stresses comparable to Integrity ${ }^{\circledR}$ provisional restoration product and greater than Jet ${ }^{\circledR}$ provisional restoration product [18] and Park et al. found no significant difference during wear testing of self-cured, milled and 3D-printed resin restorations [19]. However, these investigations were conducted on test bars [18] and prefabricated blocks [19], thereby not representing intraoral conditions.

Current studies of provisional materials' mechanical properties report flexural strength $[3,12,13,20]$ with three-point bending tests and fracture toughness [21,22] with single edge notch specimens. Furthermore, existing literature focuses on testing different self-cured, heat-cured and milled polymer-based provisional materials. Investigating the fracture strength of 3D-printed polymeric provisional materials is essential given the rapid incorporation of 3D-printing into prosthesis fabrication.

This study aims to investigate the impact of manufacture technique on the fracture strength of provisional resin 3-unit fixed dental prostheses (FDPs). Processing techniques examined include self-curing, CAD-CAM milling and 3D printing. The null hypothesis is that the fracture strength of provisional 3-unit FDPs is independent of the manufacturing process.

\section{Materials and Methods}

A metal master typodont was prepared for the mandibular left sextant typodont from titanium grade 5 (Ti 90\%, Al 6\%, V 4\%) by Panthera Dental. Two Straumann Bone Level Tapered Regular CrossFit ${ }^{\circledR}$ implant analogs (Straumann RC 025.4101; Straumann, Basel, Switzerland) were embedded at the first premolar and first molar positions with a missing second premolar (Figure 1A). Two Straumann cementable abutments were inserted at the positions of the first premolar (Straumann RC 022.4326 $\varnothing 5.0 \mathrm{~mm}$; Straumann, Basel, Switzerland) and first molar (Straumann RC $022.4336 \varnothing 6.5 \mathrm{~mm}$; Straumann, Basel, Switzerland) and a 3-unit master FDP was designed in 3Shape (3Shape Dental Software; 3Shape A/S, Copenhagen, Denmark) using the 3Shape tooth library and converted to a standard tessellation language (STL) file (Figure 2A; Supplementary File S1). The 3-unit master FDP measured $24.3 \mathrm{~mm}$ mesial-distal, $9.2 \mathrm{~mm}$ buccal-lingual and $7.3 \mathrm{~mm}$ occlusal-gingival; the second premolar pontic measured $6.7 \mathrm{~mm}$ mesial-distal, $8.0 \mathrm{~mm}$ buccal-lingual and $6.9 \mathrm{~mm}$ occlusal gingival with an occlusal table at $5.6 \times 5.3 \mathrm{~mm}^{2}$. The connection areas between the retainer and pontic are $4.3 \times 3.3 \mathrm{~mm}^{2}$ (mesial) and $3.4 \times 4.0 \mathrm{~mm}^{2}$ (distal). 

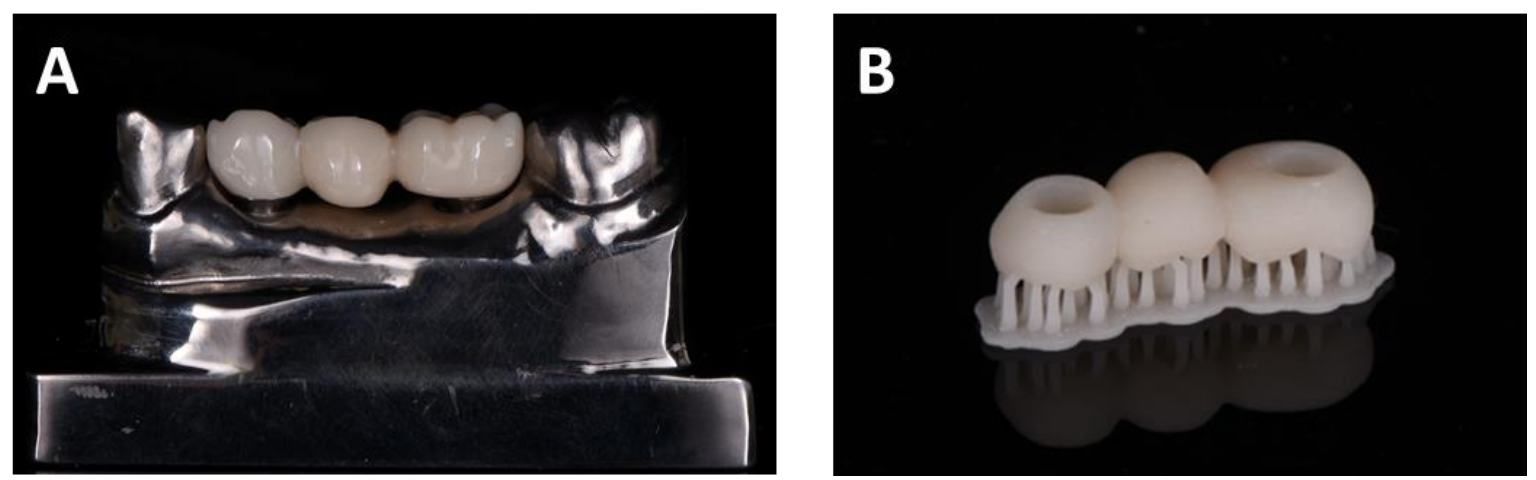

Figure 1. (A) Metal master typodont fabricated with Straumann Bone Level Tapered Regular CrossFit ${ }^{\circledR}$ implant analogs and cementable abutments at mandibular left first premolar and first molar. Self-cured poly(methyl methacrylate) provisional fixed dental prosthesis is placed on metal master typodont. (B) 3D-printed resin provisional fixed dental prosthesis with support structures.

A

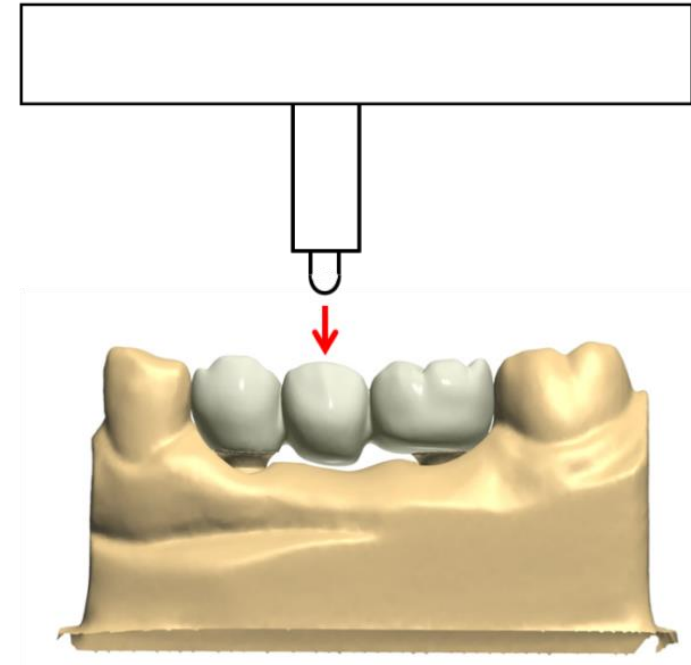

B

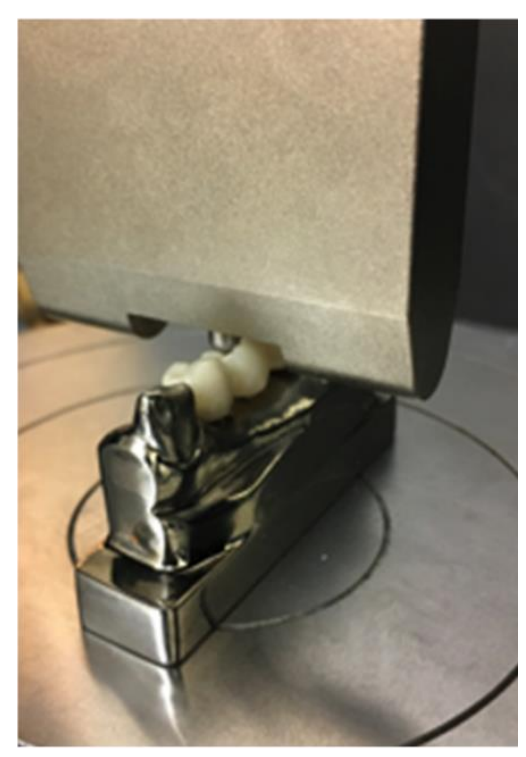

Figure 2. (A) Standard tessellation language file of 3-unit master fixed dental prosthesis designed in 3Shape by NDX H\&O Dental Laboratory to fit the metal master typodont. The connection areas between the retainer and pontic are $4.3 \times 3.3 \mathrm{~mm}^{2}$ (mesial) and $3.4 \times 4.0 \mathrm{~mm}^{2}$ (distal). Buccal and occlusal surfaces are shown. Schematic of Instron upper anvil and red vectors indicate location of applied force. (B) Metal master typodont with a provisional fixed dental prosthesis attached to the universal Instron platform. Upper anvil is positioned over the pontic occlusal surface for fracture testing.

Forty-five provisional 3-unit FDPs were fabricated with three different materials: self-cured PMMA ( $\mathrm{N}=15)$, milled PMMA from prefabricated CAD-CAM discs $(\mathrm{N}=15)$ and 3D-printed resin $(\mathrm{N}=15)$ (Figure 1). Table 1 summarizes the materials utilized during experimentation.

Table 1. Provisional resin materials and manufacturer information.

\begin{tabular}{ccccc}
\hline Type & Material & Composition & Manufacturer & Shade \\
\hline Self-cured & Jet (polymer) & Autopolymerizing PMMA & Lang Dental, Wheeling, IL, USA & A1 \\
Self-cured & Jet (monomer) & Autopolymerizing PMMA & Lang Dental, Wheeling, IL, USA & \\
Milled & Zirlux Temp & PMMA prefabricated blocks & Henry Schein, Melville, NY, USA & A1 \\
3D-printed & Freeprint Temp & Light-curing, biocompatible resin & DETAX, GmbH \& Co., DE, USA & A1 \\
\hline
\end{tabular}

For the self-cured PMMA set, PMMA (Jet; Lang Dental, Wheeling, IL, USA) and monomer were manually mixed with powder/liquid ratio (1.25:1) following the manufacturer's instructions and 
injected into a putty matrix of the FDP unit. Samples were prepared in shade A1. Samples were placed in a heat pot $(322.04 \mathrm{~K}, 124.11 \mathrm{kPa})$ for $20 \mathrm{~min}$. Samples were finished, polished and sealed with a light-curing sealant (Palaseal; Kulzer, Hanau, Germany). For the CAD-CAM PMMA set, the FDPs were milled from CAD-CAM PMMA prefabricated discs in shade A1 (Zirlux; Henry Schein, Melville, NY, USA) using a wet milling machine (Versamill; Axsys Dental Solutions, Wixom, MI, USA). The milling machine parameters were as follows: 5 axis; $6.0 \mathrm{~mm}$ shaft tool; 60,000 rpm spindle. Samples were finished, polished and sealed with a light-curing sealant (Palaseal; Kulzer, Hanau, Germany). For the 3D-printed resin set, samples were fabricated using a stereolithography-based 3D printer (MiiCraft 125; MiiCraft, Jena, Germany) with a light-curing, biocompatible resin (Freeprint Temp; DETAX GmbH \& Co. KG, Ettlingen, Germany) (Figure 1B). The printer settings were as follows: layer thickness of $50 \mu \mathrm{m}$, wavelength of $405 \mathrm{~nm}$, resin color of A1, curing time of $2.40 \mathrm{~s}$ per layer, degree orientation of $0^{\circ}$. Once the print was completed, the FDPs were soaked in 99\% isopropyl alcohol for $60 \mathrm{~s}$ and then sprayed dry with compressed air. The FDPs were placed in a second 99\% isopropyl alcohol bath for $50 \mathrm{~s}$ and then sprayed dry with compressed air. The FDPs were post-cured under UV light for $90 \mathrm{~s}$ with UV Post Curing Chamber (220 V; Paul H. Gesswein \& Co., Inc., Bridgeport, CT, USA). Samples were finished, polished and sealed with a light-curing sealant (Palaseal; Kulzer, Hanau, Germany).

Fracture strength of provisional FDPs was tested using semi-clinical experimental design at ambient laboratory conditions. The metal master typodont was fixed on the platform of a universal Instron instrument (Instron 5566 Universal Testing Machine; Instron, Norwood, MA, USA) for fracture testing (Figure 2B), and no luting agent was used to attach the FDP to the implant abutments of the master metal typodont. The dimensions of the specimens following a uniform design were visually inspected. The fit and seat of all specimens were verified with an explorer and fit checker. A stainless steel knife-edge adaptor was modified to a spherical tip (diameter $=4 \mathrm{~mm}$ ). The spherical tip was centered on the central fossa on the occlusal surface of the pontic (Figure 2). The abutment screws were torqued to $35 \mathrm{Ncm}$ prior to each experimental test and an axial load was applied at a crosshead speed of $0.5 \mathrm{~mm} / \mathrm{min}$ until fracture occurred. Maximum force at fracture was recorded (Bluehill 3; Instron, Norwood, MA, USA).

The point of fracture was visualized on the plot of extension vs. load. All statistical analysis was performed using SPSS for Mac (SPSS; IBM Corp., Armonk, NY, USA). A Shapiro-Wilk test was used to assess normality. An ANOVA was used to compare force at fracture between the three sets at significance level 0.05. Tukey post-hoc analysis was used to determine which experimental set differed.

\section{Results}

The descriptive statistics and distribution of force at FDP fracture for the three experimental sets are given in Table 2 and compared in Figure 3.

All experimental groups were normally distributed according to the Shapiro-Wilk test. Mean force at FDP fracture of 3D-printed resin differed significantly from the mean force at fracture of either self-cured $(p=0.016,95 \%$ CI $[17.86,197.91])$ or milled ( $p=0.010,95 \%$ CI $[23.83,203.88])$ PMMA and the mean forces recorded at FDP fracture of self-cured and milled PMMA showed no significant difference.

Table 2. Descriptive statistics of force at provisional fixed dental prosthesis fracture.

\begin{tabular}{cccccc}
\hline & Mean (N) & SD (N) & Median (N) & Maximum (N) & Minimum (N) \\
\hline Self-cured & 300.61 & 98.94 & 291.04 & 482.13 & 136.29 \\
Milled & 294.64 & 60.34 & 292.31 & 412.03 & 167.83 \\
3D-printed & 408.49 & 132.16 & 420.82 & 603.33 & 163.66 \\
\hline
\end{tabular}




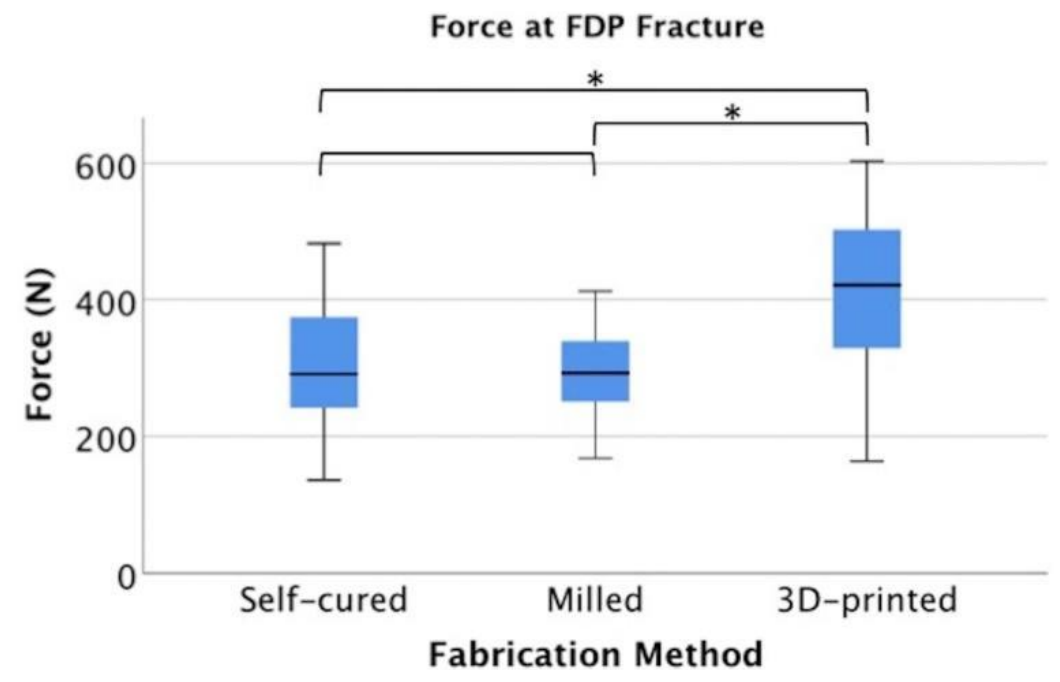

Figure 3. Force at fracture for self-cured PMMA, milled PMMA and 3D-printed resin experimental sets. $(\mathrm{N}=15)$ Shapiro-Wilk was used to test for normality. ANOVA and Tukey post-hoc tests were used to compare experimental groups at significance level 0.05. Asterisk $(*)$ indicates a significant difference.

\section{Discussion}

Our findings show that mean force at FDP fracture of 3D-printed resin is significantly greater than that of self-cured or milled PMMA. Under the conditions of this study, it can be concluded that the 3D-printed resin FDPs have more favorable fracture strength during compression than the self-cured or milled PMMA FDPs.

The fracture strength of 3D-printed resin may be explained by the increased flexural strength relative to self-cured or milled PMMA. More flexural strength will result in bending at the onset of compression, thus the 3D-printed resin provisional FDPs are less likely to fracture because bending movement absorbs initial force. The fracture strength of milled PMMA over self-cured PMMA can be attributed to the processing after initial polymerization of prefabricated PMMA discs to maximize polymerization; it is hypothesized that the higher force at fracture values are related to higher load bearing capacity of the polymer network caused by previously described effects $[1,23]$.

Data for 3D-printed prostheses have a standard deviation of $132.16 \mathrm{~N}$, while the data for self-cured and milled PMMA FDPs have standard deviations of $98.94 \mathrm{~N}$ and $60.34 \mathrm{~N}$, respectively. The greater variation in force at fracture of self-cured PMMA provisional FDPs when compared to milled PMMA provisional FDPs is logical as the self-cured PMMA is manually mixed before injection into the putty matrix; homogeneity of the provisional is not guaranteed and the material density at the point of loading may vary. It is possible that the large standard deviation of 3D-printed resin results from anisotropy and the staircase effect of printing or post-printing finishing as slight differences in curing and/or occlusal table geometry would change the force vectors during compression.

Existing studies measure flexural strength $[3,12,13,20]$, the stress in a material before it yields during flexure and fracture toughness [21,22], the stress required to propagate a crack. This study investigates fracture strength, the stress at which a sample fails via fracture. Similarly, Alt et al. reported fracture strength of direct and CAD-CAM PMMA and poly(ethyl methacrylate) (PEMA) restorations and reported the force at fracture of Cercon Base PMMA as $416.9 \mathrm{~N}$ [1], greater than the force of fracture of milled PMMA provisional FDPs in our study. However, Alt et al. stored samples in water bath for 1 day at $37^{\circ} \mathrm{C}$ [1]. Comparing fracture strength values across studies should be done with caution as the specimen design, storage conditions and testing protocol can influence quantitative results. Similar to our study, Alt concluded that CAD-CAM restorations displayed higher force at fracture than direct restorations [1].

The mechanical properties of 3D-printed restorations may be influenced by FDP design and printer specifications. The structure of the FDP affects how force propagates through the prosthesis, 
as resistance to fracture of a restoration is dependent on length of span, abutment and pontic design and material thickness [24]. Previous literature states that fracture most often occurs at the junction between the retainer and pontic [24-26]. Changing the design may impact the prosthesis' ability to withstand load.

Many printer parameters are predetermined, such as printing velocity, cure depth and cured line width; other parameters may be set by the operator: print orientation, position on the build platform and configuration of supporting structures [27]. Previous studies have investigated a correlation between print orientation and strength. Print orientation describes the direction in which the sample is printed, layer-by-layer, on the 3D printing platform [28]. The print orientation impacts printing time, as the number of layers used to fabricate varies between directions [27,28]. Printing time is a key process parameter to optimize because it relates to manufacturing cost [28]. Alharbi et al. reported that layer orientation perpendicular to load exhibits higher compressive strength than material in which the layer orientation is parallel [27]. In our experiments, the FDP samples were printed at $0^{\circ}$ orientation with the uniaxial load applied perpendicular to layers; it is possible that fabricating the samples at an angled or $90^{\circ}$ orientation would impact fracture strength.

This study shows that 3D-printed resin restorations withstand greater forces than prostheses made by conventional methods. Resins used for provisional materials are weaker after fracture and subsequent repair [5], therefore increasing fracture strength of resin provisional prostheses by incorporating 3D printing into the workflow of fabrication has potential to increase clinician productivity while creating less material waste and using less energy $[6,18]$.

The present study has limitations. Experimental conditions were in vitro and did not simulate the oral environment. Compression testing was modeled after the 3-point bending test, rather than cyclic loading which would better simulate mastication. With respect to materials, the self-cured and milled FDPs were PMMA, while the 3D-printed FDPs were methacrylate-based resin. The intent of materials selection was to use the base material in each manufacturing category, as PMMA cannot be printed by stereolithography-based printers; however, it is possible that DETAX Freeprint Temp UV methacrylate-based resin is inherently stronger than PMMA.

This study addresses the paucity of literature, but more studies are required to elucidate the mechanical properties of 3D-printed restorations. Future work will investigate wear resistance, flexural strength, fracture toughness and color stability. Cyclic loading and ageing experimentation can simulate masticatory forces and elucidate differences in wear resistance between prostheses fabricated by varied manufacturing techniques. Understanding flexural strength quantitatively will be important for 3-unit prostheses. This study's preliminary testing showed that 3D-printed resin FDPs demonstrated greater flexure than self-cured and milled PMMA FDPs, though specific vertical displacement of the pontic during fracture testing could not be recorded. Analyzing fracture toughness with single edge notch specimens will allow for value comparison across existing literature on self-cured and milled PMMA. Color stability is important to ensure esthetics and patient acceptance.

\section{Conclusions}

Mean force at provisional FDP fracture of 3D-printed resin is significantly greater than the mean force at fracture of either self-cured or milled PMMA. Additive manufacturing displays improved fracture strength of provisional prostheses and expedited delivery.

Supplementary Materials: The following are available online at http://www.mdpi.com/2673-1592/2/4/30/s1, File S1.

Author Contributions: Conceptualization, K.M.S. and J.S. and S.J.L.; methodology, K.M.S. and J.S. and C.-Y.C. and S.J.L.; formal analysis, K.M.S.; data curation, K.M.S.; writing-original draft preparation, K.M.S.; writing-review and editing, K.M.S. and J.S. and C.-Y.C. and S.J.L.; supervision, S.J.L.; funding acquisition, S.J.L. All authors have read and agreed to the published version of the manuscript.

Funding: This research received no external funding. 
Acknowledgments: The authors would like to acknowledge NDX H\&O Dental Laboratory and Cusp Dental Laboratory for fabrication of the self-cured and milled PMMA FDPs and the master typodont, respectively. We would like to thank Asa Eckert-Erdheim and the Wyss Institute at Harvard University for access to Instron instrumentation. We would like to thank Stanley Cotreau and the Harvard Physics/SEAS Instructional Machine Shop for adaptation of the Instron upper anvil.

Conflicts of Interest: The authors declare no conflict of interest.

\section{References}

1. Alt, V.; Hanning, M.; Wostmann, B.; Balkenhol, M. Fracture strength of temporary fixed partial dentures: CAD/CA versus directly fabricated restorations. Dent. Mater. 2001, 27, 339-347. [CrossRef] [PubMed]

2. Kelvin Khng, K.Y.; Ettinger, R.L.; Armstrong, S.R.; Lindquist, T.; Gratton, D.G.; Qian, F. In vitro evaluation of the marginal integrity of CAD/CAM interim crowns. J. Prosthet. Dent. 2016, 115, 617-623. [CrossRef] [PubMed]

3. Yao, J.; Li, J.; Wang, Y.; Huang, H. Comparison of the flexural strength and marginal accuracy of traditional and CAD/CAM interim materials before and after thermal cycling. J. Prosthet. Dent. 2014, 112, 649-657. [CrossRef] [PubMed]

4. Rayyan, M.M.; Aboushelib, M.; Sayed, N.M.; Ibrahim, A.; Jimbo, R. Comparison of interim restorations fabricated by CAD/CAM with those fabricated manually. J. Prosthet. Dent. 2015, 114, 414-419. [CrossRef] [PubMed]

5. Koumjian, J.H.; Nimmo, A. Evaluation of fracture resistance of resins used for provisional restorations. J. Prosthet. Dent. 1990, 64, 654-657. [CrossRef]

6. Barazanchi, A.; Li, K.C.; Al-Amleh, B.; Lyons, K.; Waddell, J.N. Additive Technology: Update on Current Materials and Applications in Dentistry. J. Prosthodont. 2017, 26, 156-163. [CrossRef]

7. Hernandez, E.P.; Oshida, Y.; Platt, J.A.; Andres, C.J.; Barco, M.T.; Brown, D.T. Mechanical properties of four methylmethacrylate-based resins for provisional fixed restorations. Bio-Med. Mater. Eng. 2004, 14, 107-122.

8. Hazelton, L.R.; Nicholls, J.I.; Brudvik, J.S.; Daly, C.H. Influence of reinforcement design on the loss of marginal seal of provisional fixed partial dentures. Int. J. Prosthodont. 1995, 8, 572-579.

9. Shillingburg, H.T.; Sather, D.A.; Wilson, E.L.; Cain, J.R.; Mitchell, D.L.; Blanco, L.J.; Kessler, J.C. Provisional Restorations. In Fundamentals of Fixed Prosthodontics; Quintessence Publishing Co, Inc.: Hanover Park, IL, USA, 2012; p. 241.

10. Gratton, D.G.; Aquilino, S.A. Interim restorations. Dent. Clin. North Am. 2004, 48, 487-497. [CrossRef] [PubMed]

11. Astudillo-Rubio, D.; Delgado-Gaete, A.; Bellot-Arcis, C.; Montiel-Company, J.M.; Pascual-Moscardo, A.; Almerich-Silla, J.M. Mechanical properties of provisional dental materials: A systematic review and meta-analysis. PLoS ONE 2018, 13, e0193162. [CrossRef]

12. Al-Dwairi, Z.N.; Tahboub, K.Y.; Baba, N.Z.; Goodacre, C.J. A Comparison of the Flexural and Impact Strengths and Flexural Modulus of CAD/CAM and Conventional Heat-Cured Polymethyl Methacrylate (PMMA). J. Prosthodont. 2018, 29, 341-349. [CrossRef]

13. Alp, G.; Murat, S.; Yilmaz, B. Comparison of Flexural Strength of Different CAD/CAM PMMA-Based Polymers. J. Prosthodont. 2019, 28, e491-e495. [CrossRef]

14. Zandinejad, A.; Methani, M.M.; Schneiderman, E.D.; Revilla-León, M.; BDS, D.M. Fracture Resistance of Additively Manufactured Zirconia Crowns when Cemented to Implant Supported Zirconia Abutments: An in vitro Study. J. Prosthodont. 2019, 28, 893-897. [CrossRef]

15. Javaid, M.; Haleem, A. Current status and applications of additive manufacturing in dentistry: A literature-based review. J. Oral Biol. Craniofacial Res. 2019, 9, 179-185. [CrossRef]

16. Bae, E.J.; Jeong, I.D.; Kim, W.C.; Kim, J.H. A comparative study of additive and subtractive manufacturing for dental restorations. J. Prosthet. Dent. 2017, 118, 187-193. [CrossRef]

17. Mai, H.N.; Lee, K.B.; Lee, D.H. Fit of interim crowns fabricated using photopolymer-jetting 3D printing. J. Prosthet. Dent. 2017, 118, 208-215. [CrossRef]

18. Tahayeri, A.; Morgan, M.; Fugolin, A.P.; Bompolaki, D.; Athirasala, A.; Pfeifer, C.S.; Ferracane, J.L.; Bertassoni, L.E. 3D printed versus conventionally cured provisional crown and bridge dental materials. Dent. Mater. 2018, 34, 192-200. [CrossRef] 
19. Park, J.M.; Ahn, J.S.; Cha, H.S.; Lee, J.H. Wear Resistance of 3D Printing Resin Material Opposing Zirconia and Metal Antagonists. Materials 2018, 11, 1043. [CrossRef]

20. Chung, S.M.; Yap, A.U.J.; Chandra, S.P.; Lim, C.T. Flexural strength of dental composite restoratives: Comparison of biaxial and three-point bending test. J. Biomed. Mater. Res. B 2004, 71B, 278-283. [CrossRef]

21. Knobloch, L.A.; Kerby, R.E.; Pulido, T.; Johnston, W.M. Relative fracture toughness of bis-acryl interim resin materials. J. Prosthet. Dent. 2011, 106, 118-125. [CrossRef]

22. Lewis, G.; Mladsi, S. Correlation between impact strength and fracture toughness of PMMA-based bone cements. Biomaterials 2000, 21, 775-781. [CrossRef]

23. Ferracane, J.L.; Condon, J.R. Post-cure heat treatments for composites: Properties and fractography. Dent. Mater. 1992, 8, 290-295. [CrossRef]

24. Kelly, J.R.; Tesk, J.A.; Sorensen, J.A. Failure of all-ceramic fixed partial dentures in vitro and in vivo: Analysis and modeling. J. Dent. Res. 1995, 74, 1253-1258. [CrossRef]

25. Oh, W.S.; Anusavice, K.J. Effect of connector design on the fracture resistance of all-ceramic fixed partial dentures. J. Prosthet. Dent. 2002, 87, 536-542. [CrossRef]

26. Sorensen, J.A.; Cruz, M.; Mito, W.T.; Raffeiner, O.; Meredith, H.R.; Foser, H.P. A clinical investigation on three-unit fixed partial dentures fabricated with a lithium disilicate glass-ceramic. Pract. Periodontics Aesthetic Dent. Ppad 1999, 11, 95-106; quiz 108.

27. Alharbi, N.; Osman, R.; Wismeijer, D. Effects of build direction on the mechanical properties of 3D-printed complete coverage interim dental restorations. J. Prosthet. Dent. 2016, 115, 760-767. [CrossRef]

28. Chacón, J.M.; Caminero, M.A.; García-Plaza, E.; Núñez, P.J. Additive manufacturing of PLA structures using fused deposition modelling: Effect of process parameters on mechanical properties and their optimal selection. Mater. Des. 2017, 124, 143-157. [CrossRef]

Publisher's Note: MDPI stays neutral with regard to jurisdictional claims in published maps and institutional affiliations.

(C) 2020 by the authors. Licensee MDPI, Basel, Switzerland. This article is an open access article distributed under the terms and conditions of the Creative Commons Attribution (CC BY) license (http://creativecommons.org/licenses/by/4.0/). 